\title{
Smart supramolecular sensing with cucurbit[n]urils: probing hydrogen bonding with SERS $\uparrow$
}

\author{
Bart de Nijs, (D) *a Marlous Kamp, (D) 'b Istvan Szabó, (D)

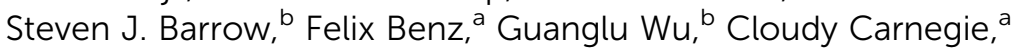 \\ Rohit Chikkaraddy, ${ }^{a}$ Wenting Wang, ${ }^{a}$ William M. Deacon, ${ }^{a}$ \\ Edina Rosta, (D) Jeremy J. Baumberg (D) *a and Oren A. Scherman (D) *b
}

Received 26th April 2017, Accepted 29th June 2017

DOI: $10.1039 / c 7 f d 00147 a$

Rigid gap nano-aggregates of Au nanoparticles formed using cucurbit[n]uril ( $\mathrm{CB}[n])$ molecules are used to investigate the competitive binding of ethanol and methanol in an aqueous environment. We show it is possible to detect as little as $0.1 \%$ methanol in water and a ten times higher affinity to methanol over ethanol, making this a useful technology for quality control in alcohol production. We demonstrate strong interaction effects in the SERS peaks, which we demonstrate are likely from the hydrogen bonding of water complexes in the vicinity of the $\mathrm{CB}[n] \mathrm{s}$.

\section{Introduction}

Surface-Enhanced Raman Scattering (SERS) has attracted wide-spread interest on account of its potential for sensing down to the single molecule level. ${ }^{1-8}$ This has led to a wealth of research on detecting trace amounts of pollutants, ${ }^{9}$ poisons ${ }^{\mathbf{1 0}}$ and explosives, ${ }^{11,12}$ achieved by plasmonic constructs such as core-shell nanoparticles ${ }^{13}$ nanostars, ${ }^{14-18}$ nanoparticle aggregates, ${ }^{19,20}$ nanowire junctions ${ }^{21-24}$ and plasmonic tips ${ }^{25-27}$ (Tip-Enhanced Raman Scattering, TERS). Recently, SERS has also been used to study reactions ${ }^{28}$ and conformational changes in molecules. ${ }^{29}$ Only a few studies have been performed where SERS is exploited to study solvent molecules as analytes, ${ }^{30,31}$ and usually only their bulk Raman signal is mentioned. ${ }^{32}$

\footnotetext{
${ }^{a}$ NanoPhotonics Centre, Cavendish Laboratory, University of Cambridge, Cambridge CB3 OHE, UK. E-mail: bd355@cam.ac.uk; jjb12@cam.ac.uk

${ }^{b}$ Department of Chemistry, Melville Laboratory for Polymer Synthesis, University of Cambridge, Cambridge CB2 1EW, UK. E-mail: oas23@cam.ac.uk

'King's College London, Department of Chemistry, London, UK

$\dagger$ Electronic supplementary information (ESI) available: Additional experimental results, additional computational details and results, supplementary references. See DOI: 10.1039/c7fd00147a
} 
Cucurbit $[n]$ urils or $\mathrm{CB}[n] \mathrm{s}$ are a family of macrocyclic molecules that have attracted increased attention on account of their excellent molecular recognition properties and high binding affinities for guest molecules. ${ }^{33}$ The ability of $\mathrm{CB}[n] \mathrm{s}$ to act as sub-nm molecular spacers to induce and control the aggregation of gold nanoparticles (AuNPs) and to subsequently bind analyte molecules at room temperature within their hydrophobic cavities is a major area of research. These simple, robust $\mathrm{CB}[n]-$ AuNP constructs have proven particularly interesting in the area of molecular sensing. ${ }^{34}$ Using SERS, it is possible to detect a wide variety of analytes which form $1: 1$ complexes within $\mathrm{CB}[7] \cdot{ }^{35}$ This host-guest chemistry is also capable of performing quantitative multiplexing of neurotransmitters in biological media such as urine. ${ }^{36}$ Moreover, on account of the strong field confinement $(>\times 100)$ within the cavities between the gold nanoparticles, analyte concentrations down to the nanomolar regime can be detected. ${ }^{36}$ Plasmonic coupling at such short distances is highly sensitive to variations in the cavity properties, making it possible to resolve thickness differences of $0.1 \mathrm{~nm}$ and observe refractive index changes from molecular filling. ${ }^{37}$

Here, we shift our attention to the detection of solvent molecules and show that $\mathrm{CB}[n]$-AuNP constructs can be used to detect the presence of methanol $(\mathrm{MeOH})$ in ethanol $(\mathrm{EtOH}) /$ water mixtures. In addition to being useful in view of public health and safety, our results show that the hydrogen bonding between $\mathrm{MeOH}$ and water and $\mathrm{EtOH}$ and water can be directly probed using plasmonic $\mathrm{CB}$ $[n]$-AuNP constructs, opening up avenues to studying the molecular dynamics of solvent molecule mixtures using SERS. With DFT calculations we explore the binding behaviour of $\mathrm{MeOH}$ and $\mathrm{EtOH}$ to $\mathrm{CB}[5]$ and $\mathrm{CB}[6]$ further and show that in addition to host-guest complexation, the direct aqueous environment is probed as well using such experimental techniques.

\section{Experimental details}

Citrate-stabilized gold nanoparticles $(60 \mathrm{~nm})$ were purchased from BBI Solutions, and used as received (and which have been previously shown to be effective). Ethanol (EtOH, absolute, $>99.8 \%$ ) and methanol $(\mathrm{MeOH}$, reagent grade) were obtained from Fisher Scientific and used as received. $\mathrm{CB}[5]$ and $\mathrm{CB}[6]$ were separated from a $\mathrm{CB}[n]$ mixture synthesised in-house using previously published procedures. ${ }^{38} \mathrm{~A} \mathrm{CB}[5]$ solution in water $[1 \mathrm{mM}]$ was bubbled with nitrogen for $30 \mathrm{~min}$. $\mathrm{CB}[6]$ is only soluble to a maximum of $200 \mathrm{mM},{ }^{39}$ therefore a $1 \mathrm{~g} \mathrm{~L}^{-1}$ suspension was prepared and bubbled with nitrogen before diluting it by a factor 50 with AuNP dispersions as described below.

Sample mixtures were prepared by mixing $\mathrm{CB}[n]$ solution, AuNP dispersion, and $\mathrm{MeOH} / \mathrm{EtOH}$ water mixtures. Typically, $20 \mu \mathrm{L}$ of the $\mathrm{CB}[n]$ solution was placed in a plastic cuvette and then $980 \mu \mathrm{L}$ gold NP dispersion was added to the $\mathrm{CB}[n]$ using an Eppendorf pipette, by which the $\mathrm{CB}[n]$ and the AuNPs were mixed. After waiting 4 min to allow for the formation of gold NP aggregates through bridging by the $\mathrm{CB}[n], 20 \mu \mathrm{L}$ of an $\mathrm{EtOH} / \mathrm{MeOH}$ mixture was added and mixed in using a $1 \mathrm{~mL}$ pipette. Alternatively, to carry out a concentration series measurement, amounts of $1 \mu \mathrm{L}$ of $\mathrm{MeOH}$ were added to the $\mathrm{CB}[n]-$ AuNP constructs repeatedly using a $10 \mu \mathrm{L}$ pipette.

SERS measurements were carried out using a Renishaw inVia Raman microscope equipped with a Plan $5 \times$ objective with a numerical aperture of 0.12 . An 
excitation wavelength of $785 \mathrm{~nm}$ was chosen in order to excite chain plasmons in the gold NP aggregates. ${ }^{\mathbf{4 0 , 4 1}}$ The laser beam entered the sample from the (open) top of the cuvette. By performing a depth series, the optimum distance of the solution from the laser, which is the location yielding the maximum number of counts, was determined. At the ideal distance the focal point of the laser is near the liquid-air interface. This results in neither the illumination path nor the collection path passing through or near the plastic of the cuvette. The spectrum from the cuvette material is known (see ESI $\dagger$ ) and no contribution from the cuvette is seen in the recorded data. Each SERS measurement was performed with an integration time of $10 \mathrm{~s}$ for 3 accumulations at full laser power $(130 \mathrm{~mW}$ over the large solution volume). Spectra were recorded in the range of $620-1730 \mathrm{~cm}^{-1}$ with a resolution of $1.1 \mathrm{~cm}^{-1}$.

\section{Results}

$\mathrm{CB}[5]$ has a hydrophobic cavity with an internal diameter of $4.4 \AA$ (see Table 1 ). $\mathrm{MeOH}^{43}$ has a diameter of $2.1 \AA$ and a kinetic diameter of $3.6 \AA$, hence the macromolecular $\mathrm{CB}[5]$ spacer could potentially sequester or sieve out single $\mathrm{MeOH}$ molecules from solution, placing them inside the plasmonic hotspots. Upon adding increasing amounts of $\mathrm{MeOH}(0-1.9 \mathrm{v} / \mathrm{v} \%)$ to the $\mathrm{CB}[5]-\mathrm{AuNP}$ constructs in water, clear spectral changes emerge with distinct peaks at $1564 \mathrm{~cm}^{-1}$ and $1604 \mathrm{~cm}^{-1}$ (Fig. 1b and c: green). Interestingly, these peaks do not correspond to bulk Raman spectra (Fig. 1c) measured for $\mathrm{MeOH}$ (black) or EtOH (red). The peaks also do not correspond to a $10 \% \mathrm{MeOH}$ water mixture (blue trace), which displays a drastically decreased Raman signal and a slightly blueshifted peak at $1030 \mathrm{~cm}^{-1}$.

The differences in signal strength and peak location demonstrate that the obtained spectra in Fig. 1b are SERS signals. A second notable change is the decrease in the $830 \mathrm{~cm}^{-1}$ peak, a characteristic peak of $\mathrm{CB}[n]$ SERS spectra, showing that the new peaks arise from some form of interaction with the $\mathrm{CB}[5]-$ AuNP constructs. Generally, quantifying such effects has proven difficult as SERS spectra suffer from inherent background fluctuations, preventing simple comparisons among peak intensities. In addition, the decrease in the peak intensity of the $\mathrm{CB}[n]$ with the addition of the analyte (here $\mathrm{MeOH}$ ) shows that the $\mathrm{CB}[5]$ peak cannot always be used as an internal standard even though the $\mathrm{CB}[5]$ concentration is kept nearly constant throughout the experiments. These issues render SERS, without supplementation, inadequate as a quantitative analytical technique.

Table 1 Structural parameters for uncomplexed CB[5]-CB[8]

\begin{tabular}{lllll}
\hline & $\mathrm{CB}[5]^{a}$ & $\mathrm{CB}[6]^{a}$ & $\mathrm{CB}[7]^{a}$ & $\mathrm{CB}[8]^{a}$ \\
\hline Portal diameter $(\AA)$ & 2.4 & 3.9 & 5.4 & 6.9 \\
Cavity diameter $(\AA)$ & 4.4 & 5.8 & 7.3 & 8.8 \\
Cavity volume $\left(\AA^{3}\right)$ & 82 & 164 & 279 & 479 \\
Outer diameter $(\AA)$ & 13.1 & 14.4 & 16.0 & 17.5 \\
Height $(\AA)$ & 9.1 & 9.1 & 9.1 & 9.1
\end{tabular}

${ }^{a}$ Adapted with permission from ref. 42, ๑2000 ACS. 


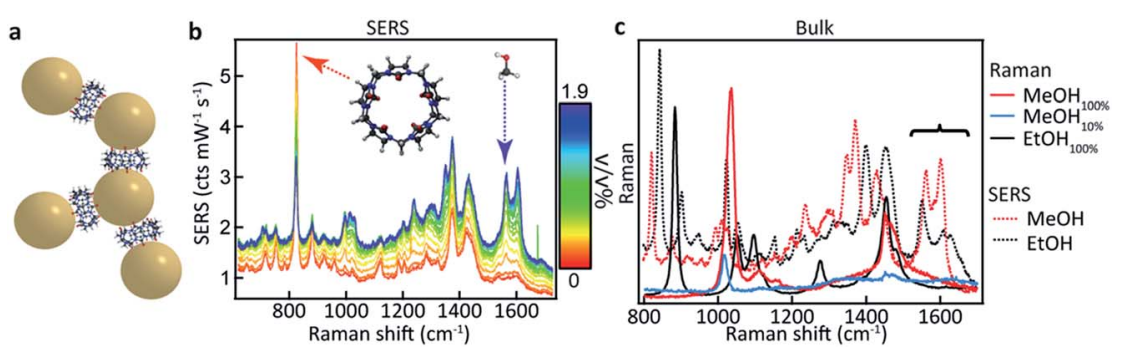

Fig. 1 SERS-based MeOH sensing in an aqueous environment. (a) Cucurbit[n]uril-gold nanoparticle construct with a $0.9 \mathrm{~nm}$ inter-particle spacing. (b) SERS spectra of CB[5]AuNP constructs showing spectral changes upon adding up to $1.9 \mathrm{v} / \mathrm{v} \% \mathrm{MeOH}$, resulting in strong peaks in the region from $1520 \mathrm{~cm}^{-1}$ to $1640 \mathrm{~cm}^{-1}$ (dashed arrow). (c) Bulk measurements of $\mathrm{MeOH}, \mathrm{MeOH}$ in water, and $\mathrm{EtOH}$ showing no peaks at $1564 \mathrm{~cm}^{-1}$ and $1604 \mathrm{~cm}^{-1}$.

Here we demonstrate that by using principal component analysis (PCA) we can accurately identify and quantify the individual analyte components and correlate SERS intensities with analyte concentrations to extract new information. PCA expresses data in a new basis set of mathematically orthogonal components in order of decreasing variability, with the aim of detecting trends in noisy, variable data such as SERS spectra. This is particularly useful when we scan the concentration $m$ of one analyte. In the case of this series of spectra, each component consists of an 'eigenspectrum' $E_{i}(\nu)$ (a set of spectral components which change together) and a weight $c_{i}$ (often known as a PCA 'score') which gives the contribution of its associated eigenspectrum to the measured spectrum. The contribution $y_{i}$ of a single component to the full spectrum at concentration $m$ is then

$$
y_{i}(m)=c_{i}(m) E_{i}
$$

Combining all the contributions yields the counts at each wavelength, reconstructing the measured spectrum

$$
y_{\mathrm{tot}}(\nu)=\sum_{i=\mathrm{I}}^{N} c_{i} E_{i}(\nu)
$$

where $y_{\text {tot }}$ is the SERS emission at wavenumber $\nu$. A practicality regarding PCA is that the physical meaning of the eigenspectra can only be attributed with additional knowledge of the experimental system.

PCA is used to exploit the change in spectra upon analyte addition to isolate the SERS contributions of the individual components from a concentration series (see Fig. 2a). The eigenspectrum of the first component obtained using PCA $\left(E_{\mathrm{I}}\right)$ closely resembles the characteristic $\mathrm{CB}[5]$ SERS and bulk Raman spectra, see Fig. S1. $\dagger$ The eigenspectrum of the second major component $\left(E_{\mathrm{II}}\right)$ is attributed to $\mathrm{MeOH}$ since a steady increase in its corresponding weight is observed with the addition of analyte (Fig. 2b-d).

However, $E_{\mathrm{II}}$ does not only contain $\mathrm{MeOH}$ lines, but also shows a reduction (negative peaks) in the CB lines (Fig. 2a, II). This clearly shows the existence of some chemical interaction between the components. As a result of the basic properties of PCA, since variability is expressed as a weight about the origin of the 

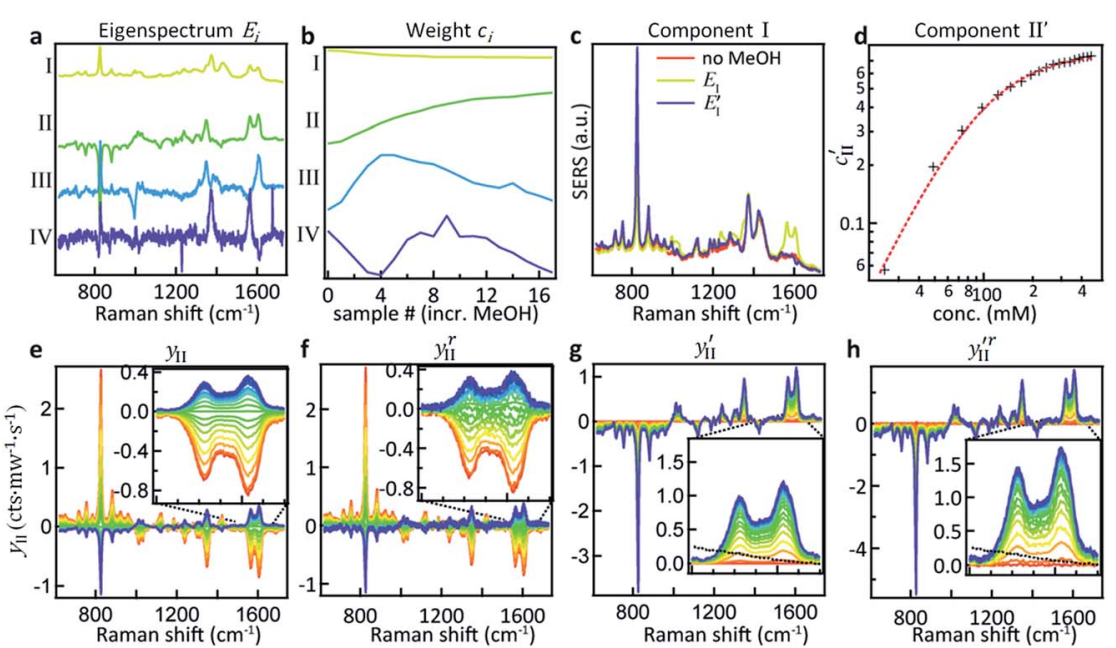

Fig. 2 Principal component analysis of spectral changes in $\mathrm{CB}[5]-A u N P$ aggregates upon the addition of $\mathrm{MeOH}$. (a) The first four component eigenspectra $E_{i}$. (b) Weights $c_{i}$ for each of the components showing their variation upon the addition of $\mathrm{MeOH}$ from 0-500 mM. (c) Comparison of $E_{1}, E_{1}^{\prime}$ and a pure $\mathrm{CB}[5]$ spectrum without $\mathrm{MeOH}$. (d) The modified PCA II weight $C^{\prime}{ }_{\|}$plotted against $\mathrm{MeOH}$ concentration shows a clear correlation. (e) Plot showing $E_{\|}$multiplied by its weight highlighting the changes upon addition of $\mathrm{MeOH}$. (f) Reconstituted change in PCA II, plotted by subtracting $y_{1, I I I-V I}$ from the raw dataset. (g and h) Same plots as (e and f) but with the weight offset by its value at $m=0 \mathrm{v} / \mathrm{v} \% \mathrm{MeOH}$, showing the reconstructed spectral changes for PCA II. The dotted line in the inset is the bulk Raman signal of $\mathrm{MeOH}$ in this region.

principal axes, $c_{\mathrm{II}}(0)$ has a negative value at $0 \% \mathrm{MeOH}$, and $E_{\mathrm{I}}$ (light blue trace in Fig. 2c) does not represent a clean $\mathrm{CB}[5]$ spectrum. This contribution can be removed from $E_{\mathrm{I}}$ by extracting the fraction of $E_{\mathrm{II}}$ from $E_{\mathrm{I}}$ at $0 \% \mathrm{MeOH}$ using

$$
E_{\mathrm{I}}^{\prime}=E_{\mathrm{I}}-E_{\mathrm{II}} \times \frac{c_{\mathrm{II}}(0 \%)}{c_{\mathrm{I}}(0 \%)}
$$

This essentially moves the data set along PC axis II, making the lowest weight value [corresponding to no $\mathrm{MeOH}$ added] equal to 0 . This results in $E_{\mathrm{I}}^{\prime}$ (dark blue in Fig. 2c) closely matching the $\mathrm{CB}[5]$ spectrum ('no $\mathrm{MeOH}$ ', red) without $E_{\mathrm{II}}$. Similarly we define

$$
c_{\mathrm{II}}^{\prime}(m)=c_{\mathrm{II}}(m)-c_{\mathrm{I}}(m) \times \frac{c_{\mathrm{II}}(0 \%)}{c_{\mathrm{I}}(0 \%)}
$$

now resulting in only positive weight values for the modified second component. Plotting this $c_{\text {II }}^{\prime}$ versus the added analyte concentration gives a clear saturation curve (Fig. 2d) which is well fit by the Hill-Langmuir equation,

$$
c^{\prime}{ }_{\mathrm{II}}=A_{\mathrm{II}}\left[1+\left(K_{\mathrm{D}} /[\mathrm{MeOH}]\right)^{n}\right]^{-1}
$$

where $K_{\mathrm{D}}$ is the dissociation constant (here $140 \mathrm{mM}$ ), $n=2.0$ is the Hill coefficient which is here markedly higher than 1 indicating a cooperative binding 
mechanism for the $\mathrm{MeOH}$, and the maximum $A_{\mathrm{II}}=0.73$ is the saturated component value.

The components extracted using PCA can be exploited to visualise, without noise present, the spectral changes happening as $\mathrm{MeOH}$ is added (Fig. 2e). The effect of dimensionality reduction (with only 6 components being significant and taken into account) can be observed by taking the dataset and subtracting the contributions from all the components (I-VI) except II:

$$
y_{\mathrm{II}}^{\mathrm{r}}(m) \simeq y_{\text {tot }}(m)-\sum_{i=\mathrm{I}, \quad}^{\mathrm{VI}} c_{i \neq \mathrm{II}}(m) E_{i}
$$

reconstituting the contribution of the second component (Fig. 2f). The good match between $y_{\mathrm{II}}$ and $y_{\mathrm{II}}^{\mathrm{r}}$ in Fig. 2e and f shows that individual spectra can indeed be suitably reconstructed from these components, even when interacting.

The effect of shifting the data origin to match $m=0$ can be seen when using the modified $c_{\text {II }}^{\prime}$ and $E_{\mathrm{I}}^{\prime}$, with ${y^{\prime}}_{i}={c^{\prime}}_{i} E^{\prime}{ }_{i}$ and the equivalent of eqn (6) to calculate $y_{\mathrm{II}}^{\mathrm{r}}$. This gives the induced changes on adding $\mathrm{MeOH}$ concentrations of up to $2 \%$ (Fig. $2 \mathrm{~g}$ and $\mathrm{h}$ ) and confirms that plotting the reconstituted eigenspectra yields spectral changes characteristic for the added analyte.

These more complex spectral changes with increasing analyte concentration clearly show that the SERS peaks are correlated with $\mathrm{MeOH}$ concentration, even though the isolated spectral change (Fig. 2h) shows minimal resemblance to the bulk MeOH Raman spectrum (black dashed). This radical difference between the bulk (Raman) and SERS spectra of $\mathrm{MeOH}$ suggests that binding effects or other strong molecular interactions between the $\mathrm{MeOH}$ and either the gold, the $\mathrm{CB}[5]$, or other moieties in the system play an important role in the detection. We thus explored this interaction further.

Comparing EtOH with $\mathrm{MeOH}$ as an analyte, similar peaks are observed throughout the SERS spectra (Fig. 3a) but with a distinct exception in the region around $1550 \mathrm{~cm}^{-1}$ (Fig. 3b). While a similar doublet of peaks is observed here for both $\mathrm{EtOH}$ and $\mathrm{MeOH}$ with one peak located at $1604 \mathrm{~cm}^{-1}$, for EtOH a shifted peak at $1539 \mathrm{~cm}^{-1}$ is observed compared to the $\mathrm{MeOH}$ peak at $1564 \mathrm{~cm}^{-1}$. The similarity between these spectra suggests similar but non-identical interactions between the $\mathrm{CB}[5]$ and these analytes.
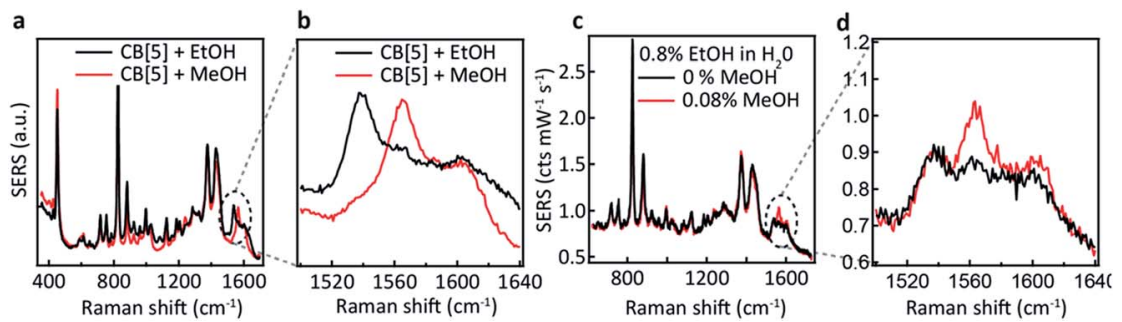

Fig. $3 \mathrm{CB}[5]-A u N P$ SERS demonstrating $\mathrm{MeOH}$ and EtOH detection. (a) SERS spectra of $\mathrm{EtOH}$ (red) and $\mathrm{MeOH}$ (black). (b) Spectral region of interest showing distinct differences in the peak position for $\mathrm{EtOH}\left(1540 \mathrm{~cm}^{-1}\right)$ and $\mathrm{MeOH}\left(1564 \mathrm{~cm}^{-1}\right)$. (c) Detection of $4 \mathrm{v} / \mathrm{v} \%$ $\mathrm{MeOH}$ (red) or no $\mathrm{MeOH}$ (black), when combined with a mixture of $40 \mathrm{v} / \mathrm{v} \% \mathrm{EtOH}$ : water. (d) Highlighted region showing a clear peak at $1564 \mathrm{~cm}^{-1}$. 
We now explore the ability to combine EtOH and MeOH SERS detection, which is crucial for their application to consumer alcohol contamination. We also investigate a hypothesis that the interaction behaviour originates from sizespecific encapsulation of methanol inside $\mathrm{CB}[5]$. Two mixtures $(20 \mu \mathrm{L})$ are added as analytes to the $\mathrm{CB}[5]$-AuNP sensing aggregates $(980 \mu \mathrm{L})$, both containing $40 \mathrm{v} / \mathrm{v} \% \mathrm{EtOH}$ in water, which in one case is further spiked with $4 \mathrm{v} / \mathrm{v} \% \mathrm{MeOH}$. For the non-spiked sample, a weaker version of the two ethanol peaks is observed (Fig. 3c and d). For the spiked sample, an additional sharp peak at $1564 \mathrm{~cm}^{-1}$ is seen along with a small increase in the $1604 \mathrm{~cm}^{-1}$ peak, which corresponds to the $\mathrm{MeOH}$ SERS spectrum previously determined. Notice that the final concentration of EtOH in the $1 \mathrm{~mL}$ measurement volume is $0.8 \mathrm{v} / \mathrm{v} \%$, and that we are able to measure a final concentration as low as $0.08 \mathrm{v} / \mathrm{v} \%$. Whilst the total added amount of $\mathrm{MeOH}$ is 10 times lower than the amount of EtOH added, a comparably intense SERS peak is observed ( $\sim 0.1 \mathrm{cts}$ per $\mathrm{mW}$ per s each). This suggests a preference for $\mathrm{MeOH}-\mathrm{CB}[5]$ over EtOH-CB[5].

To support this, we calculated the association energies using quantum chemical calculations at the DFT B3LYP-Def2-TZVP-D3 level (see ESI4 Table S1 $\dagger$ ) as well as biased umbrella sampling MD free energy calculations (Fig. 4a and b). Since there is a $2.9 \mathrm{kcal} \mathrm{mol}^{-1}$ free energy gain for the $\mathrm{MeOH}$ to reside in the $\mathrm{CB}[5]$ cavity based on the MD simulations and 0.7 or $2.4 \mathrm{kcal} \mathrm{mol}^{-1}$ gain based on the DFT calculations using SMD or PCM solvent models, respectively, inclusion should be favourable. We also calculated that the $\mathrm{MeOH}$ molecule has to overcome a 7.7 and $2.0 \mathrm{kcal} \mathrm{mol}^{-1}$ free energy barrier to pass through the carbonyl portals into the $\mathrm{CB}[5]$ and $\mathrm{CB}[6]$ cavities, respectively along the trajectory marked (Fig. 4a). This kinetic barrier is in line with observed inclusion results for methane
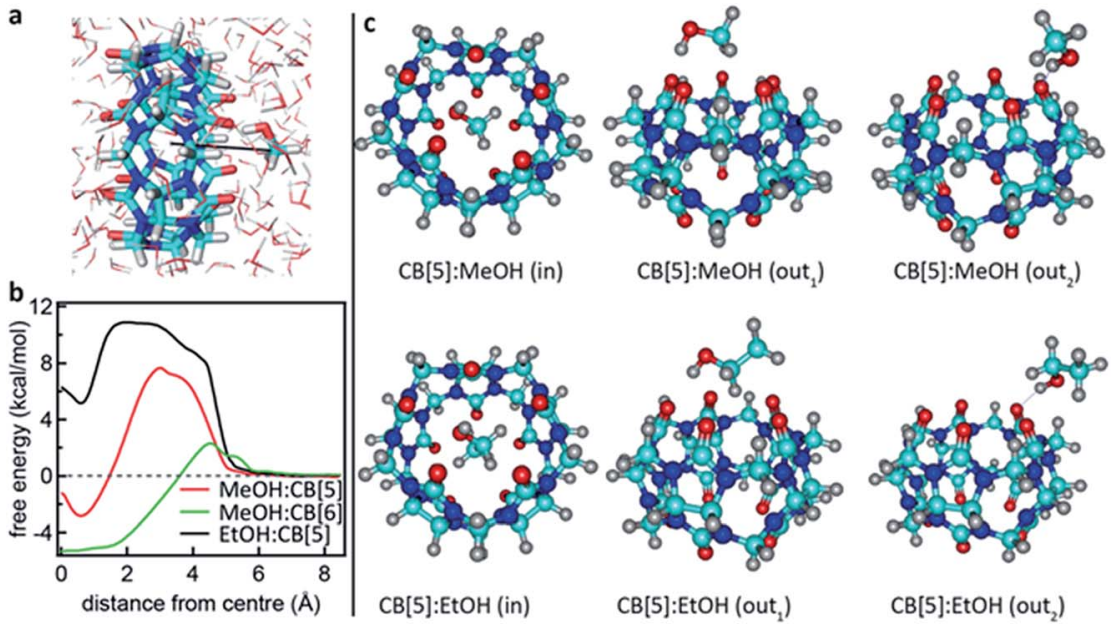

Fig. 4 Calculated interaction between $\mathrm{CB}[5]$ and $\mathrm{MeOH}$ or EtOH. (a) Rendering showing the $\mathrm{MeOH}$ being moved into the $\mathrm{CB}[5]$ cavity in an aqueous environment. (b) Free energy profiles for $\mathrm{MeOH}$ and $\mathrm{EtOH}$ ligands passing through the carbonyl portals of $\mathrm{CB}[5]$ and $\mathrm{CB}[6]$ and into the molecular cage. (c) Renderings of geometry-optimized alternative complexations between $\mathrm{CB}[5]$ and $\mathrm{MeOH}$ or $\mathrm{EtOH}$ (see also the Computational details in the ESI $\uparrow$ ). 
in $\mathrm{CB}[5]$ where heating to $80{ }^{\circ} \mathrm{C}$ is required for sequestration to occur. ${ }^{44}$ Nevertheless, our experiments clearly point at a preferential molecular interaction for $\mathrm{MeOH}-\mathrm{CB}[5]$ with respect to $\mathrm{EtOH}-\mathrm{CB}[5]$. To explore the possibility that the outside of $\mathrm{CB}[5]$ preferentially interacts with $\mathrm{MeOH}$ over EtOH, we calculated the association free energy for $\mathrm{CB}[5]$ complexed with $\mathrm{MeOH}$ and $\mathrm{EtOH}$ more comprehensively (Table S1 $\dagger$ ). Only a marginally lower free energy is found for the $\mathrm{MeOH}$, which is not enough to explain the observed preferential interaction. Since the calculations indicate that inclusion of $\mathrm{EtOH}$ by $\mathrm{CB}[5]$ is highly unfavourable, these experiments suggest that in such systems the outside of the $\mathrm{CB}[5]$ is probed as well since EtOH is still readily detected. Interestingly, the fitted dissociation constant $\left(K_{\mathrm{D}}\right)$ for $\mathrm{EtOH}-\mathrm{CB}[5]$ is lower than the $K_{\mathrm{D}}$ fitted for $\mathrm{MeOH}-$ $\mathrm{CB}[5]$ (ESI: Table S2†).

It has been shown in the literature that $\mathrm{MeOH}$ inclusion by decamethyl-CB[5] does occur in the presence of ammonia. ${ }^{45}$ Ammonia is absent in the current experiments, but sodium, a citrate counter-ion, has a similar interaction with the portals $^{46}$ and is present in the system. It is clear that subtle solvent effects influence this binding behaviour as bubbling nitrogen through the $\mathrm{CB}[n]$ solution, or varying between different batches of $\mathrm{CB}[5]$ (not shown here), results in different but consistent spectral changes, demonstrating a strong interaction of the analyte with its environment.

What is not yet explained are the positions of the SERS peaks that do not match any of the bulk Raman signals. This shows again that the analyte interacts with either the $\mathrm{CB}[n]$ or other chemical moieties in its direct environment. To identify the potential nature of these SERS peaks, vibrational spectra were calculated for each of the $\mathrm{CB}[5]: \mathrm{MeOH}$ and $\mathrm{CB}[5]$ :EtOH binding geometries presented in Fig. 4, and for other complexes of $\mathrm{CB}[5]$ with competitive guests potentially present in the ambient system $\left(\mathrm{H}_{2} \mathrm{O}, \mathrm{N}_{2}, \mathrm{O}_{2}\right)$. These are compared to the vibrational spectra of hydrogen-bonded complexes (denoted by a colon) of $\mathrm{MeOH}$ and $\mathrm{EtOH}$ with water. All three configurations of $\mathrm{CB}[5]$ : $\mathrm{MeOH}$ and $\mathrm{CB}[5]$ :EtOH show additional peaks only in the region of $1450 \mathrm{~cm}^{-1}$ to $1500 \mathrm{~cm}^{-1}$ (Fig. $5 \mathrm{a}, \mathrm{b}$, e and f) but no peaks near those observed at $1539 \mathrm{~cm}^{-1}, 1564 \mathrm{~cm}^{-1}$, or $1604 \mathrm{~cm}^{-1}$. While no clear change in this $1500-1620 \mathrm{~cm}^{-1}$ band is observed for nitrogen or water inside CB [5], placing oxygen in the molecular cavity is predicted to give a clear line at 1585 $\mathrm{cm}^{-1}$ (Fig. $5 \mathrm{c}$ and $\mathrm{g}$ ) directly from the oxygen molecule, which is thus one possibility.

However even without $\mathrm{CB}[5]$ present, calculated vibrational spectra for hydrogen-bonded complexes between $\mathrm{MeOH}$ and water $\left(\mathrm{MeOH}: \mathrm{H}_{2} \mathrm{O}\right)$, between EtOH and water (EtOH: $\left.\mathrm{H}_{2} \mathrm{O}\right)$, and between two water molecules $\left(\mathrm{H}_{2} \mathrm{O}: \mathrm{H}_{2} \mathrm{O}\right)$ also exhibit peaks in the same region (Fig. $5 \mathrm{~d}$ and $\mathrm{h}$ ). These are at $1576 \mathrm{~cm}^{-1}$ for water/ $\mathrm{H}_{2} \mathrm{O}: \mathrm{H}_{2} \mathrm{O} / \mathrm{MeOH}: \mathrm{H}_{2} \mathrm{O}$, at $1593 \mathrm{~cm}^{-1}$ for $\mathrm{H}_{2} \mathrm{O}: \mathrm{H}_{2} \mathrm{O}$, and at $1597 \mathrm{~cm}^{-1}$ for EtOH: $\mathrm{H}_{2} \mathrm{O}$. Since it is unlikely that the oxygen concentration increases when $\mathrm{MeOH}$ is added (although this cannot be excluded), this suggests it is the hydrogen bonding between the analyte and water in or near the $\mathrm{CB}[5]$ cavity that is probed by SERS. Strong or arrested hydrogen bonding is expected, as otherwise broader peaks would be observed, hinting at a local 'solid' phase with temporarily arrested dynamics.

Using $\mathrm{CB}[6]$ instead of $\mathrm{CB}[5]$ results in peak positions at $1539 \mathrm{~cm}^{-1}$ and $1604 \mathrm{~cm}^{-1}$ (see ESI Fig. S2 $\dagger$ ), matching the dominant peaks obtained with EtOH and $\mathrm{CB}[5]$, further supporting the idea that the peaks are a result of hydrogen 

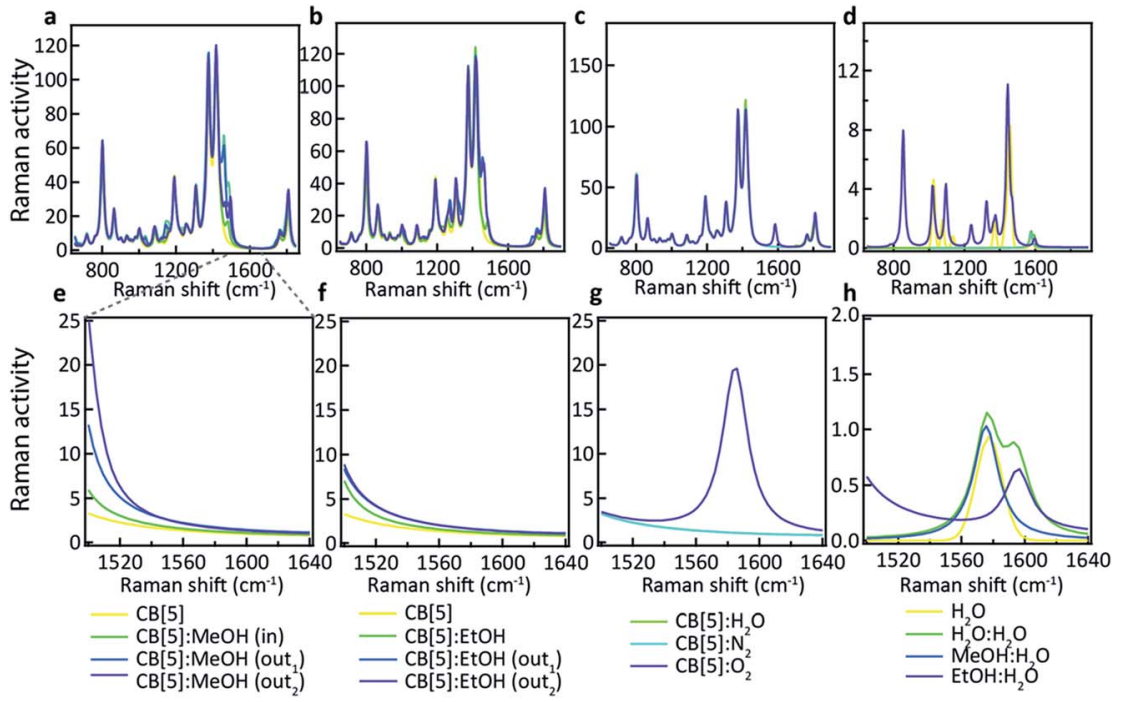

Fig. 5 Calculated vibrational spectra for plausible molecular binding geometries with $\mathrm{CB}[5]$ present in the probed system. Positioned in and around the macromolecular structure: (a) $\mathrm{MeOH}$, (b) EtOH, (c) water, nitrogen, and oxygen. (d) Water molecules hydrogen-bonded with water, $\mathrm{MeOH}$ and $\mathrm{EtOH}$, without $\mathrm{CB}[5]$. (e-h) Highlighted range of interest showing that only oxygen and hydrogen-bonded water have peaks at these wavenumbers.

bonding. The exact configuration of the $\mathrm{MeOH}: \mathrm{H}_{2} \mathrm{O}$ and $\mathrm{EtOH}: \mathrm{H}_{2} \mathrm{O}$ complexation is not yet clear, and nor is the observed influence of the direct environment, but the use presented here of $\mathrm{CB}[n]$-AuNP constructs in combination with $\mathrm{MeOH}$ and EtOH appears to be a powerful method of probing the behaviour of water in confined spaces. Considering the immense sensitivity achievable with SERS (down to single molecules) these advances open up new avenues in probing molecular mechanics and solvent-solvent interactions with an unprecedented level of detail. We also demonstrate that such a system can be practically used for the detection of $\mathrm{MeOH}$ in alcoholic liquids providing a low-cost potential means of screening for health risks in consumer products.

\section{Conclusions}

Using the $\mathrm{CB}[5]$ and $\mathrm{CB}[6]$-based self-assembly of gold nanoparticles into sensing aggregates, we demonstrated SERS-based detection of as little as $0.1 \mathrm{v} / \mathrm{v} \%$ of methanol in water. We also demonstrated that even in ethanol/water mixtures, $4 \mathrm{v} / \mathrm{v} \%$ methanol (the upper safe consumption limit) can be distinctly detected. We show that principal component analysis can be used to isolate the methanol contribution to the SERS spectra. Our data imply that $\mathrm{CB}[n]$ molecules either sequester or strongly interact with $\mathrm{MeOH}$ and to a lesser extent EtOH, and the obtained signals arise from interactions with water around the molecular spacers. We conclude that this provides a powerful test bed for probing molecular dynamics at the nanoscale. 


\section{Acknowledgements}

We acknowledge financial support from the UK's Engineering and Physical Sciences Research Council (grants EP/L027151/1, EP/K028510/1, EP/L015978/1, and EP/N020669/1) and the European Research Council (grants LINASS 320503, MSCA-IF-2015-EF SPARCLEs 7020005 and MSCA-IF-2014-EF NANOSPHERE, 658360). RC acknowledges support from the Dr Manmohan Singh scholarship from St. John's College. FB acknowledges support from the Winton Programme for the Physics of Sustainability. CC acknowledges support from the UK National Physical Laboratories. GW thanks the Leverhulme Trust (Natural material innovation for sustainable living) for their support.

\section{Notes and references}

1 S. Nie and S. R. Emory, Science, 1997, 275, 1102.

2 N. P. W. Pieczonka and R. F. Aroca, Chem. Soc. Rev., 2008, 37, 946.

3 C. J. L. Constantino, T. Lemma, P. A. Antunes and R. F. Aroca, Anal. Chem., 2001, 73, 3674.

4 Q. Si, Phys. Rev. Lett., 1997, 78, 1667.

5 J. N. Anker, W. P. Hall, O. Lyandres, N. C. Shah, J. Zhao and R. P. Van Duyne, Nat. Mater., 2008, 7, 442.

6 S. Lal, S. Link and N. J. Halas, Nat. Photonics, 2007, 1, 641.

7 M. D. Sonntag, J. M. Klingsporn, A. B. Zrimsek, B. Sharma, L. K. Ruvuna and R. P. Van Duyne, Chem. Soc. Rev., 2014, 43, 1230.

8 K. Kneipp, H. Kneipp, I. Itzkan, R. R. Dasari and M. S. Feld, J. Phys.: Condens. Matter, 2002, 14, R597.

9 J. Ofner, et al., Anal. Chem., 2016, 88, 9766.

10 M. Mulvihill, A. Tao, K. Benjauthrit, J. Arnold and P. Yang, Angew. Chem., Int. Ed., 2008, 47, 6456.

11 K. Kneipp, et al., Spectrochim. Acta, Part A, 1995, 51, 2171.

12 Z. Xu, J. Hao, W. Braida, D. Strickland, F. Li and X. Meng, Langmuir, 2011, 27, 13773.

13 J. F. Li, et al., Nature, 2010, 464, 392.

14 C. G. Khoury and T. Vo-Dinh, J. Phys. Chem. C, 2008, 112, 18849.

15 W. Niu, Y. A. A. Chua, W. Zhang, H. Huang and X. Lu, J. Am. Chem. Soc., 2015, 137, 10460.

16 S. He, M. W. C. Kang, F. J. Khan, E. K. M. Tan, M. A. Reyes and J. C. Y. Kah, J. Opt., 2015, 17, 114013.

17 A. Childs, E. Vinogradova, F. Ruiz-Zepeda, J. J. Velazquez- Salazar and M. JoseYacaman, J. Raman Spectrosc., 2016, 47, 651.

18 S. Harmsen, et al., Sci. Transl. Med., 2015, 7, 271 ra7.

19 D. V. Voronine, et al., Sci. Rep., 2012, 2, 891.

20 P. P. Patra, R. Chikkaraddy, R. P. N. Tripathi, A. Dasgupta and G. V. P. Kumar, Nat. Commun., 2014, 5, 4357.

21 R. Dasari and F. P. Zamborini, Anal. Chem., 2016, 88, 675.

22 J. M. Baik, S. J. Lee and M. Moskovits, Nano Lett., 2009, 9, 672.

23 Y. Fang, H. Wei, F. Hao, P. Nordlander and H. Xu, Nano Lett., 2009, 9, 2049.

24 R. Chikkaraddy, D. Singh and G. V. P. Kumar, Appl. Phys. Lett., 2012, 100, 043108. 
25 B. Pettinger, P. Schambach, C. J. Villagómez and N. Scott, Annu. Rev. Phys. Chem., 2012, 63, 379.

26 E. Bailo and V. Deckert, Chem. Soc. Rev., 2008, 37, 921.

27 J. Stadler, T. Schmid and R. Zenobi, Nano Lett., 2010, 10, 4514.

28 S. Dick, et al., Adv. Mater., 2016, 28, 5705.

29 D. Kurouski, M. Mattei and R. P. Van Duyne, Nano Lett., 2015, 15, 7956.

30 Z. Wang, Z. Tian, D. Han and F. Gu, ACS Appl. Mater. Interfaces, 2016, 8, 5466.

31 Y. Kihara, H. Asami and J.-Y. Kohno, J. Phys. Chem. B, 2017, 121(17), 4538.

32 S. O. Kucheyev, J. R. Hayes, J. Biener, T. Huser, C. E. Talley and A. V. Hamza, Appl. Phys. Lett., 2006, 89, 053102.

33 S. J. Barrow, S. Kasera, M. J. Rowland, J. del Barrio and O. A. Scherman, Chem. Rev., 2015, 115, 12320.

34 R. Chikkaraddy, B. de Nijs, F. Benz, S. J. Barrow, O. A. Scherman, E. Rosta, A. Demetriadou, P. Fox, O. Hess and J. J. Baumberg, Nature, 2016, 535, 127.

35 D. O. Sigle, S. Kasera, L. O. Herrmann, A. Palma, B. de Nijs, F. Benz, S. Mahajan, J. J. Baumberg and O. A. Scherman, J. Phys. Chem. Lett., 2016, 7, 704.

36 S. Kasera, L. O. Herrmann, J. D. Barrio, J. J. Baumberg and O. A. Scherman, Sci. Rep., 2014, 4, 6785.

37 B. de Nijs, R. W. Bowman, L. O. Herrmann, F. Benz, S. J. Barrow, J. Mertens, D. O. Sigle, R. Chikkaraddy, A. Eiden, A. Ferrari, O. A. Scherman and J. J. Baumberg, Faraday Discuss., 2015, 178, 185.

38 A. Day, A. P. Arnold, R. J. Blanch and B. J. Snushall, Org. Chem., 2001, 66, 8094.

39 J. Zhao, H.-J. Kim, J. Oh, S.-Y. Kim, J. W. Lee, S. Sakamoto, K. Yamaguchi and K. Kim, Angew. Chem., 2001, 113, 4363; Angew. Chem., Int. Ed., 2001, 40, 4233. 40 R. W. Taylor, T. C. Lee, O. A. Scherman, R. Esteban, J. Aizpurua, F. M. Huang, J. J. Baumberg and S. Mahajan, ACS Nano, 2011, 5, 3878.

41 R. W. Taylor, R. Esteban, S. Mahajan, J. Aizpurua and J. J. Baumberg, J. Phys. Chem. C, 2016, 120, 10512.

42 J. Kim, et al., J. Am. Chem. Soc., 2000, 122, 540.

43 H. Wu, Q. Gong, D. H. Olson and J. Li, Chem. Rev., 2012, 112, 836.

44 M. Yuji, A. Kazuaki and I. Takahiko, Angew. Chem., 2002, 41, 3020.

45 K. A. Kellersberger, J. D. Anderson, S. M. Ward, K. E. Krakowiak and D. V. Dearden, J. Am. Chem. Soc., 2001, 123, 11316.

46 X.-L. Ni, et al., Chem. Soc. Rev., 2013, 42, 9480. 